\title{
A new measurement tool for characterization of superconducting rf accelerator cavities using high-performance LTS SQUIDs
}

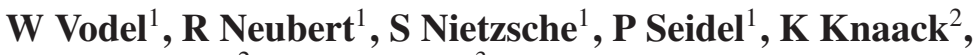 \\ K Wittenburg ${ }^{2}$ and A Peters ${ }^{3}$ \\ ${ }^{1}$ Friedrich-Schiller-University Jena, Helmholtzweg 5, 07743 Jena, Germany \\ ${ }^{2}$ DESY Hamburg, Germany \\ ${ }^{3}$ Heidelberger Ionenstrahl-Therapiezentrum, Heidelberg, Germany \\ E-mail: wolfgang.vodel@uni-jena.de,klaus.knaack@desy.de and \\ andreas.peters@med.uni-heidelberg.de
}

Received 7 June 2007, in final form 27 August 2007

Published 18 October 2007

Online at stacks.iop.org/SUST/20/S393

\begin{abstract}
This paper presents a new system to measure very low currents in an accelerator environment, using a cryogenic current comparator (CCC). In principle a CCC is a conventional current transformer using the high-performance SQUID technology to sense the magnetic fields caused by the beam current. Since the system is sensitive on a pA level, it is an optimum device to detect dark currents of superconducting cavities. The system presented here is designed for the test facilities of the superconducting accelerator modules for the European XFEL at the Deutsches Elektronen-Synchrotron (DESY) in Hamburg. Measurements in a quiet environment showed that an intrinsic noise level of the CCC of $40 \mathrm{pA} \mathrm{Hz}^{-1 / 2}$ could be achieved.

(Some figures in this article are in colour only in the electronic version)
\end{abstract}

\section{Introduction}

Due to the development of the TESLA technology [1], superconducting cavities have increasingly high cavity gradients. In addition to the quality factor $Q$, an important criterion for good performance is a low rate of field emission. The so-called dark current consists of particles emitted by field emission, which are captured by the accelerating fields of the cavity. Since this current can be emitted at arbitrary locations in the accelerator, dark current does not fit in energy, and thus gets lost in focusing elements close to its origin. This results in additional cryogenic loss and activation of components. Therefore, dark current is often the parameter that puts practical limits on the cavity performance.

A further increase in the gradients is technically possible by developing the preparation procedures. In order to do this, or to guarantee a given performance during a larger production series, field emission or dark current has to be controlled during the fabrication process. The device presented here provides the necessary resolution and bandwidth to measure on the pA level. The CCC device takes advantage of the existing cryogenic environment; thus the requirement for cryogenic temperatures is not an additional complication.

The linear accelerator technology, based on superconducting L-band $(1.3 \mathrm{GHz})$ cavities, is currently under study at DESY [1]. The XFEL will be equipped with a total of 116 superconducting acceleration modules, each containing eight '9-cell cavities' using high purity niobium material. A gradient of $23.4 \mathrm{MV} \mathrm{m}^{-1}$ is required for a so-called superstructure arrangement of couples of 9-cell cavities. Higher gradients up to the physical limit at $50 \mathrm{MV} \mathrm{m}^{-1}$ are desired. The dark current, due to emission of electrons in these high-gradient fields, is an unwanted particle source. Two issues are of main concern.

(1) Thermal load. An emitted electron from the cavity surface follows a path along the electric field lines, and will 
most probably hit somewhere else inside the cavity. This leads to an additional thermal load in the cryostat, which has to be absorbed by the liquid-helium refrigerator.

(2) Propagating dark current. If the energy gain is sufficient, the electrons will generate secondary particles when hitting the cavity wall, which in turn may also generate further secondaries. In the following avalanche process some electrons may pass through the iris of the cavity cell and will be further accelerated. In this case, if on average more than one electron passes the complete FODO (focus/defocus lattice) cell, the dark current along the linear accelerator (LINAC) will grow exponentially.

Theoretical studies [2] show that the second case seems to be the more critical one. It limits the acceptable dark current on the beam pipe 'exit' of a TESLA 9-cell cavity to approximately $50 \mathrm{nA}$. Therefore the mass production of high-gradient cavities with minimum field emission requires a precise, reliable measurement of the dark current in absolute values. The apparatus presented here senses dark currents in the $\mathrm{nA}$ range. It is based on the cryogenic current comparator (CCC) principle, which includes a highly sensitive LTS SQUID system as the magnetic field sensor. Furthermore, the set-up contains a Faraday cup and will be housed in the cryostat of the CHECHIA cavity test stand and mounted at a temperature of $1.8 \mathrm{~K}$.

\section{Requirements for dark current measurement apparatus}

Electrons can leave the niobium cavity material if the force of the applied external electric field is higher than the bounding forces inside the crystal structure. The highest field gradients occur at corners, spikes or other discontinuities, due to imperfections of the cavity shape. Another potential field emitter is due to any kind of imperfection on the crystal matter, such as grain boundaries, inclusion of 'foreign' contaminants (microparticles of, for example, In, $\mathrm{Fe}, \mathrm{Cr}, \mathrm{Si}, \mathrm{Cu}$ ) and material inhomogeneity. At these imperfections the bounding forces are reduced and electrons are emitted under the applied high electromagnetic fields [3]. The inner surfaces of the superconducting cavities undergo a series of special treatments to minimize these effects. A reliable, absolute measurement of the dark current allows the comparison of different processing methods and a quality control in the future mass production.

The XFEL will be operated in a pulse mode with up to $10 \mathrm{~Hz}$ repetition rate. The $1.3 \mathrm{GHz}$ rf pulse duration is $650 \mu \mathrm{s}$ flat top. During this time the dark current is present and has to be measured. Therefore a bandwidth of $10 \mathrm{kHz}$ for the dark current instrument is sufficient. As field emission is a statistical process, the electrons leave the cavity at both ends of the beam pipe. Thus, half of the dark current exits at each side, and it has to be measured at one side only. With the $1.3 \mathrm{GHz}$ rf applied, we expect that the dark current will have a strong amplitude modulation at this frequency. This frequency has to be carefully rejected from the instrument electronics to ensure its proper operation and to avoid a malfunction of the SQUID. This was done by the use of careful rf shielding, appropriate filtering of all leads feeding to the SQUID input coil, and the low-pass characteristic of the transformer used.

The use of a cryogenic current comparator as dark current sensor has some important advantages:

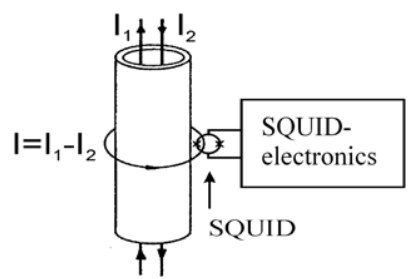

Figure 1. Simplified scheme of an LTS SQUID-based cryogenic current comparator.

- measurement of the absolute value of the dark current,

- independence of the electron trajectories,

- accurate absolute calibration with an additional wire loop, and

- extremely high resolution.

The required working temperature of $4.2 \mathrm{~K}$ (boiling temperature of liquid helium) for the apparatus is already provided by the CHECHIA test stand for cooling the niobium cavities. In order to enable the CCC to measure the magnetic field of the dark current only, effective shielding against external magnetic fields has to be realized.

\section{The cryogenic current comparator (CCC)}

In principle, the $\mathrm{CCC}$ comprises three main components (see figure 1):

- the superconducting pick-up coil,

- the highly effective superconducting shield, and

- the high-performance LTS SQUID system.

The CCC, first developed by Harvey in 1972 [4], is a nondestructive method to compare two currents $I_{1}, I_{2}$ (see figure 1) with high precision using a meander-shaped flux transducer. Thus only the magnetic field component, which is proportional to the current in the wires, will be sensed by the pick-up coil. All other field components are strongly suppressed. In our apparatus the signal current induced in the pick-up coil is fed into the input coil of a high-performance DC SQUID.

The design of the CCC for measurements of dark currents has been realized by collaboration between DESY Hamburg, Jena University, and GSI Darmstadt. The prototype apparatus will be placed in the CHECHIA cavity test stand and operated at a temperature of $4.2 \mathrm{~K}$.

\subsection{Pick-up coil}

A single-turn pick-up coil is formed as a superconducting niobium toroid with a slot around the circumference. It contains a Vitrovac 6025-F core (Vacuumschmelze $\mathrm{GmbH}$, Hanau, Germany) providing a high permeability of about 30000 at liquid-helium temperatures [5]. According to our experience, $6025-\mathrm{F}$ cores give the lowest noise level in comparison to other materials tested. The material inhomogeneity of the core is averaged by complete encapsulation of a toroidal niobium coil. 
A new measurement tool for characterization of superconducting rf accelerator cavities using high-performance LTS SQUIDs

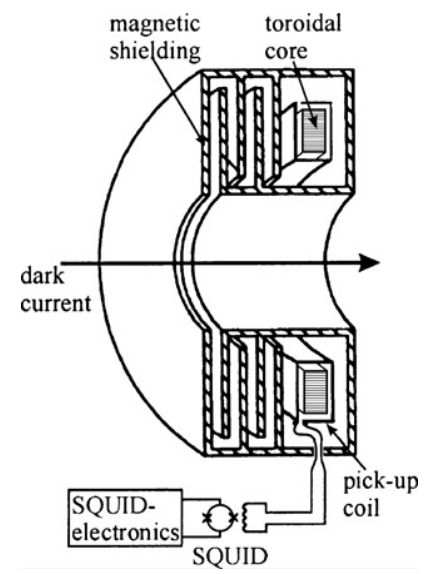

Figure 2. Simplified schematic view of the magnetic shielding, the toroidal pick-up coil, and the SQUID.

\subsection{Superconductive shields}

The resolution of the CCC is reduced if the toroidal pick-up coil operates in the presence of external disturbing magnetic fields. In practice, external fields are unavoidable; therefore an extremely effective shielding has to be applied. A circular meander ('ring cavities') shielding structure (see figure 2) allows only the azimuthally magnetic field component of the dark current to pass, while the non-azimuthal field components are strongly attenuated.

The attenuation characteristics of CCC shields have been studied analytically in great detail [6-8]. When applied to the shielding of the TESLA CCC, an attenuation factor of approximately $120 \mathrm{~dB}$ for transverse, non-azimuthal magnetic field components is estimated. This result is based on the superposition of the analytic results for the different shielding substructures, in this case coaxial cylinders and 'ring cavities' (as described in detail in [9]).

\subsection{SQUID measurement system}

The key component of the CCC is the high-performance DC SQUID system developed and manufactured at Jena University. The SQUID sensor UJ 111 [10] is designed in a gradiometric configuration and based on $\mathrm{Nb}-\mathrm{NbO}_{x}-\mathrm{Pb} / \mathrm{In} / \mathrm{Au}$ Josephson tunnel junctions with dimensions of $3 \mu \mathrm{m} \times 3 \mu \mathrm{m}$. The SQUID electronics consists of the low-noise preamplifier and the SQUID control unit. The low source impedance of the SQUID (about $1 \Omega$ ) is stepped up to the optimal impedance of the preamplifier by a resonant transformer. The DC bias and flux modulation current (modulation frequency $307 \mathrm{kHz}$ ) are fed into the SQUID via voltage-controlled current sources situated in the preamplifier and the controller, respectively. An optimal choice of bias and flux modulation point for the SQUID system was found to be a white flux spectral density of $2 \times 10^{-6} \Phi_{0} \mathrm{~Hz}^{-1 / 2}$. This flux noise corresponds to an equivalent current noise through the input coil of $0.9 \mathrm{pA} \mathrm{Hz}^{-1 / 2}$, an effective energy factor of $543 h$ ( $h$ : Planck's constant), and an energy resolution of $3.6 \times 10^{-31} \mathrm{~J} \mathrm{~Hz}^{-1}$ [10].

In a DC coupled feedback loop, the field of the dark current to be measured is compensated at the SQUID by an external magnetic field generated by the attached electronics.

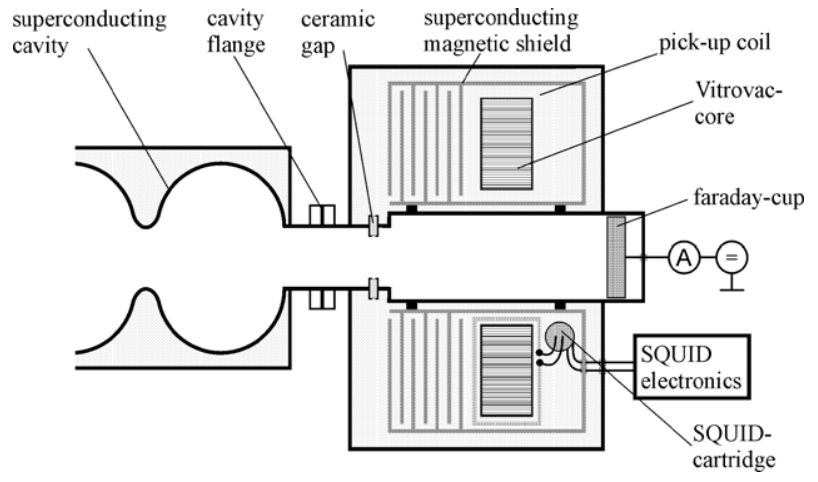

Figure 3. Schematic view of the CHECHIA's CCC.

Due to the superconductivity of all leads in the input circuitry (pick-up coil, transformer, SQUID input coil) the CCC is able to detect even DC currents. For an optimum coupling between the single-turn toroidal pick-up coil $(40 \mu \mathrm{H})$ and the SQUID input a matching transformer is necessary. The overall current sensitivity of the CCC was calculated to be $175 \mathrm{nA} / \Phi_{0}$.

Using a modulation frequency of $307 \mathrm{kHz}$ the measurement system provides an overall bandwidth of $20 \mathrm{kHz}$ (signal level $1 \Phi_{0}$ ) or $70 \mathrm{kHz}$ (signal level $0.1 \Phi_{0}$ ). Thus, it will be possible to characterize the pulse shape of the dark current beam (300 $\mu$ s rise time, $950 \mu$ s flattop, $300 \mu$ s fall time, $10 \mathrm{~Hz}$ repetition rate) which is dominated by the rf structure applied to the cavities (see also figure 6).

\subsection{Faraday cup}

Because the energy of dark current electrons is relatively small at CHECHIA, the design includes a Faraday cup to provide a second measurement system for comparison. We installed the Faraday cup at the end of the cavity vacuum chamber. The readout electronics will measure the current to ground.

The simplified scheme of the main components of the CHECHIA's CCC is shown in figure 3. Later configurations for complete module tests in the test stand will not allow simple Faraday cups because of the much higher energy (some $100 \mathrm{MeV}$ ) of the dark current.

\section{Results}

Test measurements of the completed pick-up coil with the special cabling and feed-throughs at $4.2 \mathrm{~K}$ were successfully carried out in a wide-neck cryostat at the cryogenic laboratory of Jena University (see figure 4). A current generator was used as a signal source to simulate the expected dark electron beam pulses. By supplying the calibration coil with a calibrated current pulse the current sensitivity of the CCC was found to be $200 \mathrm{nA} / \Phi_{0}$, which is in a good agreement with the design value of $175 \mathrm{nA} / \Phi_{0}$.

Figure 5 presents the calibration procedure: the upper curve is the test pulse signal of $170 \mathrm{nA}$ and the lower curve the SQUID output signal with an amplitude of $0.85 \Phi_{0}$ and a repetition rate of $1 \mathrm{~Hz}$.

Figure 6 shows a plot of the simulated beam signal generated by a programmable current generator with the 


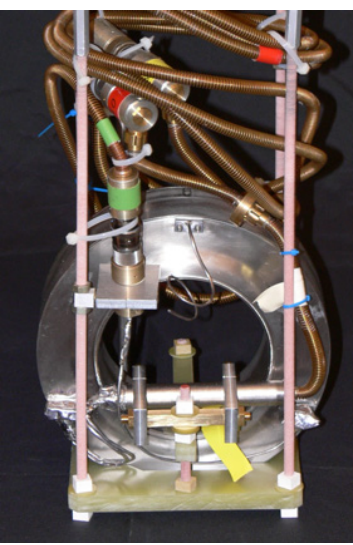

Figure 4. $\mathrm{Nb}$ pick-up coil prepared for preliminary tests at $4.2 \mathrm{~K}$ in a liquid-helium wide-neck cryostat.

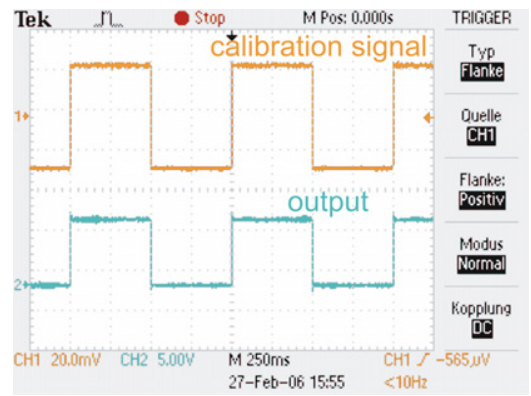

Figure 5. Calibration signal (upper curve) and SQUID output signal (lower curve). See text for details.

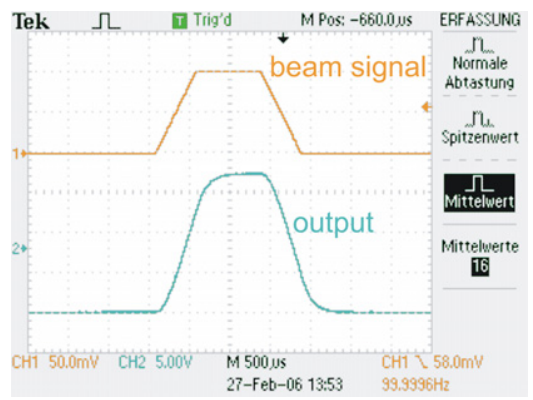

Figure 6. Simulated beam pulse (upper curve, $300 \mu$ s rise time, $950 \mu$ s flattop, $300 \mu$ s fall time) and SQUID output signal (lower curve).

amplitude of $340 \mathrm{nA}$. The response amplitude of the SQUID system was $1.7 \Phi_{0}$, corresponding to a current sensitivity of $200 \mathrm{nA} / \Phi_{0}$.

The spectral flux noise density of the system in the frequency range between 1 and $1600 \mathrm{~Hz}$ was measured using an HP spectrum analyser in the laboratory at Jena University, and a level of $2 \times 10^{-4} \Phi_{0} \mathrm{~Hz}^{-1 / 2}$ was observed (see figure 7). For these measurements a low-pass filter with a cut-off frequency of $1 \mathrm{kHz}$ was used. This flux noise level corresponds to a noise-limited current resolution of the system of $40 \mathrm{pA} \mathrm{Hz}^{-1 / 2}$, which is significantly better than required. It should be pointed out that the current resolution of the final system is not limited by the intrinsic noise of the DC SQUID.

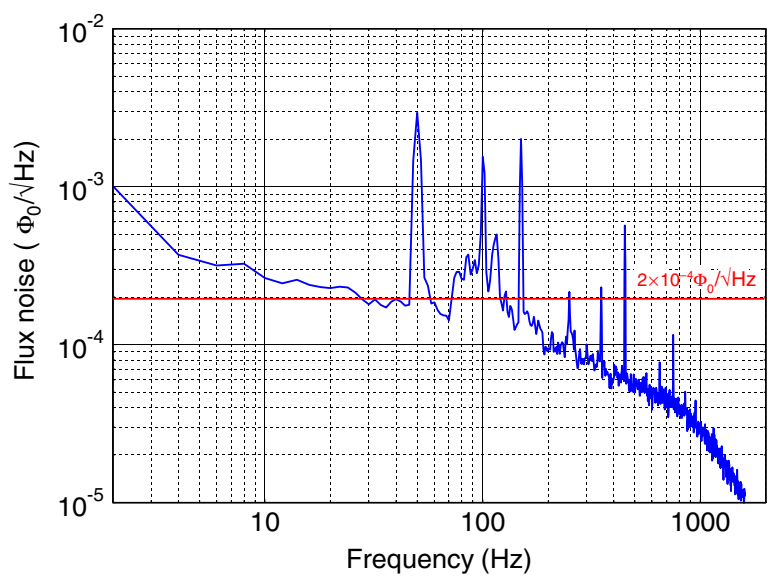

Figure 7. Noise spectrum of the CCC within a bandwidth from 1 to $1600 \mathrm{~Hz}$. Low-pass filter: $1 \mathrm{kHz}$.

The main noise sources are above all the additional noise contribution of the Vitrovac 6025-F core and environmental noise due to the non-perfect superconducting shielding.

As a result of the rough measurement conditions at DESY, a noise-limited current resolution of the CCC of $500 \mathrm{pA} \mathrm{Hz}^{-1 / 2}$ was achieved. This is primarily a result of the external electrical disturbances and mechanical vibrations caused by numerous machines and other equipment of the test facility.

Long-term measurements of the output voltage of the CCC to detect flux drifts, caused for instance by temperature drifts of the core material (Vitrovac 6025-F), showed a sufficient small drift of $<2 \times 10^{-5} \Phi_{0} \mathrm{~s}^{-1}$.

\section{Conclusion}

The mechanical construction of the CHECHIA CCC is complete. Tests of the manufacturing of critical components, above all the meander-shaped niobium shielding and the CCC's housing, were successfully carried out. The SQUID electronics including special cabling and feed throughs are ready for installation at DESY.

The completed pick-up coil with connected SQUID system were tested in a wide-neck liquid-helium cryostat at 4.2 K. By supplying the calibration coil with a calibrated current pulse, the current system sensitivity of the CCC could be measured, and a value of $200 \mathrm{nA} / \Phi_{0}$ was found.

In spite of the rough measurement conditions at DESY a noise-limited current resolution of the $\mathrm{CCC}$ of $500 \mathrm{pA} \mathrm{Hz}^{-1 / 2}$ was achieved. Measurements in a quiet environment in the low-temperature laboratory at Jena University showed that this value could be improved by more than one order of magnitude, down to a noise level of $40 \mathrm{pA} \mathrm{Hz}^{-1 / 2}$.

Long-term measurements of the output voltage of the CCC showed that the drift of the whole system is negligibly small and appears in the range of $<2 \times 10^{-5} \Phi_{0} \mathrm{~s}^{-1}$.

The complete CCC is now ready for use, and the final commissioning of the apparatus in the CHECHIA test stand is planned within the next months. The start of the dark electron beam measurements to test the superconductive accelerator $\mathrm{rf}$ cavities is planned for autumn 2007. 
A new measurement tool for characterization of superconducting rf accelerator cavities using high-performance LTS SQUIDs

\section{Acknowledgments}

This work was supported in part by the Deutsches Elektronen Synchrotron (DESY) Hamburg, Germany. The authors wish to thank R Nawrodt and A Steppke for their support in performing measurements and computer work. We also thank L Saenger as well as the company pro-beam AG, Burg, Germany, for their expert assistance in manufacturing most of the mechanical parts for the CCC. We are very grateful to I Martin for helpful discussions.

\section{References}

[1] Brinkmann R 1996 TESLA Technical Design Report
[2] Brinkmann R 2002 Dark Current Issues; TESLA Collab. Meeting (CEA Saclay)

[3] Werner G R et al 2001 PAC 01: Proc. Part. Acc. Conf. pp 1071-3

[4] Harvey I K 1972 A precise low temperature dc ratio transformer Rev. Sci. Instrum. $\mathbf{4 3} 1626$

[5] Datasheet 'Toroidal strip-wound cores of VITROVAC $6025 \mathrm{~F}$ ' and private communication from Vacuumschmelze Hanau, Germany 2002

[6] Grohmann K et al 1976 Cryogenics 16 423-9

[7] Grohmann K et al 1976 Cryogenics 16 601-5

[8] Grohmann K and Hechtfischer D 1977 Cryogenics 17 579-81

[9] Gutmann P and Bachmair H 1989 Superconducting Quantum Electronics ed V Kose (Berlin: Springer) pp 255-9

[10] Vodel W and Mäkiniemi K 1992 An ultra low noise SQUID system for biomagnetic research Meas. Sci. Technol. 3 1155-60 